To the Editors:

\title{
A situation analysis of diabetic eye care service delivery in health care institutions of the Western Province of Sri Lanka
}

\author{
P N Piyasena ${ }^{1}$, G V S Murthy ${ }^{2}$ \\ Ceylon Medical Journal 2017; 62:205-206 \\ DOI: http://doi.org/10.4038/cmj.v62i3.8527
}

There is a diabetes epidemic in most parts of the world [1]. Diabetic retinopathy affects the most productive, working age population of a country, which results in significant economic impact [2]. Many studies have shown that proper diabetes control, screening for diabetic retinopathy and early identification and treatment of complications will reduce progression of diabetic retinopathy. Prevalence of diabetes in the Western Province is $18.6 \%$, (95\% CI $15.8-21.5 \%)$ among those aged more than 18 years [3]. This is the highest prevalence in Sri Lanka. There is no scientific evidence on the current situation of diabetic retinopathy service delivery in this province. The primary objective of this study was to conduct a situation analysis of diabetic eye care services in health care institutions of the Western Province of Sri Lanka. Ethics approval was obtained from the National Eye Hospital, Colombo and the Research Ethics Committee of the London School of Hygiene and Tropical Medicine.

This was a descriptive cross sectional survey conducted from $15^{\text {th }}$ June to $26^{\text {th }}$ July 2014 in the Western Province of Sri Lanka. All health care institutions, where an ophthalmologist or a vitreo retinal surgeon was available were included in the survey. We collected data on human resources, infrastructure and diabetic retinopathy service delivery using a validated questionnaire. Cleaned and finalised data were transferred into STATA/IC $13^{\circledR}$ (version 2) analytical package for detailed analysis.
There were 51 institutions providing specialized eye care services at various levels in the Western Province. Forty three institutions provided permission to conduct the survey (Response rate $84.3 \%)$. Nine $(17.6 \%)$ provided tertiary care of which five were public funded teaching hospitals and four were private multispecialty hospitals. Twenty five $(49 \%)$ were secondary hospitals which included 11 public funded general and base hospitals and 14 private hospitals with inward facilities. There were $17(33.3 \%)$ primary level institutes, all were private hospitals without inward facilities. Thirty three $(64.7 \%)$ institutions were situated in the Colombo District $11(21.6 \%)$ in Gampaha District and 7 $(13.7 \%)$ in the Kalutara District. Thirty five $(68.6 \%)$ were private institutions and 16 (31.4\%) were public funded institutions. Twenty nine (82.9\%) institutions from the private sector and 14 (87.5\%) from the public sector participated in the survey.

All institutions were assessed for availability of diabetic retinopathy services and their output. There was no systematic diabetic retinopathy screening program in the Western Province. Patients undergo opportunistic screening (mydriatic biomicroscopy) by consultant ophthalmologists when they present to an eye care unit. There were 703,575 total patient clinic visits over a one year period in the Western province. The largest number 353,707 $(50.3 \%)$ visited secondary level institutions. A total of 135,819 opportunistic diabetic retinopathy screening visits were recorded over a one year period

\footnotetext{
${ }^{I}$ National Eye Hospital, Sri Lanka.

${ }^{2}$ Public Health Eye Care and Disability International Centre for Eye Health Clinical Research Department, London School of Hygiene and Tropical Medicine, London, United Kingdom.

Correspondence: PNP, E-mail: <Prabhath.nishantha@gmail.com>. Received 23 September 2016 and revised version accepted 9 August 2017.
} 
(May 2013 to May 2014), of which the highest number $96,784(71.3 \%)$ was in the Colombo District and the lowest number $12,722(9.4 \%)$ was in the Kalutara District. Of the screening visits 66,086 $(48.7 \%))$ were conducted in secondary level institutions and 63,008 (46.4\%) were in tertiary care centres. Majority, $81911(60.3 \%)$ of the diabetic retinopathy screening was done in the public sector.

The number of laser sessions for diabetic retinopathy performed in the one year prior to the survey was 13,553 , of which $10,949(80.8 \%)$ were done in institutions in the Colombo District. Out of the total number of laser sessions 8,551 (63.1\%) were done in tertiary care institutions. The private sector had conducted 6,862 (50.6\%) ) laser sessions. AntiVEGF intra-vitreal injections for diabetic retinopathy were given to 4,520 patients and 1,205 diabetic related pars plana vitrectomies were done in the Western Province.

The Western Province of Sri Lanka currently faces many challenges in developing eye care services. Resource allocation and expansion of service delivery is not comparable with these changes. Lack of data is a major disadvantage when lobbying for expansion of eye care services and planning for interventions. According to ophthalmologists, in recent years, many working age people present with sight threatening pathologies due to diabetic retinopathy and there are long waiting lists for diabetic retinopathy related major surgeries.

In all three districts the screening of diabetic patients is opportunistic. According to the estimated prevalence of diabetes mellitus and diabetic retinopathy, there should be 806,789 diabetic patient screening visits per year in the province. Our findings show a huge unmet need of 670,970 clinic visits for diabetic retinopathy screening. There were only 13,553 diabetic laser sessions per year in the province. Diabetic retinopathy burden calculation showed that at least 124,243 laser sessions are required for the province. This means that there is an unmet gap of 110,690 laser procedures per year and only about a tenth of the required sessions are done in the province. Additionally, long waiting lists for diabetes related major vitrectomy surgeries shows that there are a large number of people with advanced diabetic retinopathy.

This study had several limitations. This survey did not reflect the trend of diabetic retinopathy services over time. Some service providers of eye care (refraction service providers, eye clinics under medical practitioners) were excluded from the study since these services did not have a board certified ophthalmologists or a vitreo retinal surgeon. It is possible that the numbers recorded include those residing outside the province.

In conclusion this study estimates that there is a 670,970 patient visits per year gap in service provision in diabetic retinopathy screening in the Western Province of Sri Lanka. The number undergoing laser treatment is far less than the estimated number needing this service. The Western Province of Sri Lanka should develop a systematic screening program for diabetic retinopathy, with emphasis on policy development, mid-level human resource development and vitreo retinal infrastructure development in order to bridge the gap in service delivery.

\section{Acknowledgements}

All individuals and institutions that
supported in conducting the survey are acknowledged.

\section{Funding}

This project was financially supported by Sear Family Foundation, United Kingdom through a student research project grant coordinated through the International Centre for Eye Health, London, WC1E 7HT, United Kingdom.

\section{Conflicts of interest}

There are no conflicts of interest.

\section{References}

1. Whiting DR1, Guariguata L, Weil C, Shaw J. IDF diabetes atlas: global estimates of the prevalence of diabetes for 2011 and 2030. Diabetes Res Clin Pract 2011; 94: 311-21.

2. Patel MC. The socioeconomic impact of diabetic retinopathy. Retinal Physician 2009 http://www.retinalphysician.com/articleviewer.a spx?articleID-103459 (accessed on Mar 14, 2014).

3. Katulanda P, Rathnapala D, Sheriff R, Matthews D . Province and ethnic specific prevalence of diabetes among Sri Lankan adults. Sri Lanka J Diabetes Endocrinol Metab 2012; 1: 2-7.

This is an open-access article distributed under the terms of the Creative Commons Attribution License, which permits unrestricted use, distribution, and reproduction in any medium, provided the original author and source are credited. 